original papers

\title{
Does a limited nurse triage service reduce junior doctor psychiatric on-call workload?
}

\author{
AIMS AND METHOD \\ To investigate whether nurse triage \\ might reduce junior doctors' on-call \\ workloads in a general adult psy- \\ chiatry in-patient unit, we measured \\ changes in workload after the intro- \\ duction of a limited (overnight and \\ weekend mornings) nurse triage \\ service on the unit, comparing pre- \\ triage work levels with levels 1 year \\ later. This time frame allowed the \\ new service to settle in, and
}

\author{
controlled for seasonal variations in \\ workload.

\section{RESULTS} \\ The number of 'work episodes' went \\ up, especially during the night shifts, \\ but the average length of each \\ episode went down (both statistically \\ significant, $P<0.001)$. The net result \\ was a slight increase in workload in \\ terms of total time spent dealing
}

\begin{abstract}
with episodes, most notably at night.

\section{CLINICAL IMPLICATIONS}

A limited overnight nurse triage service was ineffective on its own as a means of reducing the out-of-hours workload of junior doctors, and even slightly increased it. A more comprehensive triage service, with a greater range of alternatives to admission, might have had a different result.
\end{abstract}

In the past decade, considerable effort has been put into reducing junior doctors' workload; this has been driven by diverse but converging forces and more recently by impending legal deadlines. The 'New Deal' (National Health Service Management Executive, 1991) attempted to reduce working hours, further enforced by 'punitive' pay-banding systems for onerous rotas in 2000 (Department of Health, 2000). More recently, further impetus has come from the European Working Time Directive (Council Directive, 1993) and the interpretations of the European Court of Justice ruling (Judgment of 3 October 2000) on what constitutes working hours. These, along with perceived stress levels associated with on-call work, have led to innovative ways of trying to reduce the workload of on-call junior medical staff in a broad range of specialties (Cass et al, 2003), while maintaining quality of service provision, or ideally improving it. These have included changes in shift patterns, use of non-medical practitioners and new service models. Locally, changes in delivery of mental health services are also likely to have had an effect on psychiatric junior doctors' on-call work, with the introduction of assertive outreach, crisis intervention and home treatment teams. Nationally, the government is running 20 pilot projects throughout the country across the specialties, looking at ways to reduce workload to meet the demands of the new European legislation. Two of these projects involve mental health provision, one of which hopes to use non-medical 'mental health practitioners'.

When our psychiatric unit introduced a limited nurseled triage service for on-call work in 2001, we took the opportunity to measure junior doctor on-call workload before the introduction, and again 1 year later. This time frame allowed us to compare levels after the service had had plenty of time to settle in, and also controlled for any seasonal variation in workload. The aim of the service was twofold: to try to improve service provision (shorter waiting times for patients, more efficient use of services, less tired junior doctors) and to try to move closer to meeting the impending demands for reduction in junior doctors' working hours. More by chance than design, there was no other significant change in service provision over this period.

\section{Method}

\section{Setting}

The study took place in a general adult and old-age psychiatric unit, serving a mixed urban and rural population of 270000 persons. The unit was attached to a district general hospital with an accident and emergency department, which sees 74000 patients annually, of whom approximately 100 are admitted to psychiatric wards. One junior doctor (senior house officer) on call provided an emergency service to the psychiatry wards and the rest of the hospital, including the accident and emergency department. During the day, there was a nurse-led liaison service (restricted to cases of deliberate self-harm) for patients in the accident and emergency department and the medical and surgical wards, but outside these hours such duties were covered by the on-call doctor. There was no home treatment or crisis intervention service.

The new triage nurses, who were experienced, senior staff, had other service demands in the form of nursing management duties (overall supervision of the unit), and these took priority over triage if they clashed. Unfortunately, we did not specifically measure the frequency of this occasional unavailability of the triage nurse, or the number of episodes passed on to the doctor because of it, but the authors' experience was that this was not common. The triage service was provided by one of the nurses, from $21.00 \mathrm{~h}$ to $07.00 \mathrm{~h}$ each day and from 09.00 to $13.00 \mathrm{~h}$ at weekends (i.e. $78 \mathrm{~h}$ per week). The nurses took all calls that would 
normally have gone to the on-call junior doctor; both from within the hospital and from outside. They assessed patients first, using their judgement and experience to decide what they could deal with, and what would be referred on to the junior doctor. Junior doctors were encouraged not to repeat any work already performed by the triage nurse.

\section{Measuring workload}

We could find no published study that measured psychiatric junior doctors' on-call workloads, so we developed our own data collection form (copies available from the author upon request), testing several prototypes before arriving at the final version, which was designed for ease of use. To optimise data collection, the form was filled out in 'real time' as the shift progressed, and was contained in the on-call doctor information handbook, kept with the doctor at all times.

For simplicity, we used 'number of episodes of work' and 'length of episodes' as proxy measures of workload, allowing us to combine them to give an overall measure of time worked for each shift. Length of each episode was estimated by the doctor, and placed into time bands as follows: 'short', 0-20 min; 'medium', 20-60 min; 'long', 60-120 min; 'very long', 120 min or more. For the purposes of calculating average length of episodes, the middle of each time band was used as a representative figure: $10 \mathrm{~min}, 40 \mathrm{~min}, 90 \mathrm{~min}$ and $150 \mathrm{~min}$, respectively. This arrangement was felt to provide the easiest method of data collection, albeit at the expense of uneven time increments for analysis, and potentially losing the increased precision that smaller time bands would have given. We also identified the source of each episode, allowing us to monitor changes in referral patterns (to be reported separately). All regular doctors on the rota (one in seven on call) were involved from the conception of the study, and agreed to take part (short-term locums were excluded for practical reasons). To ensure consistency, a protocol was developed, by consensus, as to what constituted an 'episode'. The data were analysed using the Statistical Package for the Social Sciences (SPSS, 1999).

\section{Results}

The sample size was 205 pre-triage shifts (over 4 months) and 90 post-triage shifts (over 2 months, same mid-point (July), 1 year on). Table 1 shows the average number of episodes and their average length for each shift involving triage nurses, along with the combined estimate of total time spent working per shift (obtained by multiplying these figures). Data were normally distributed, allowing parametric tests to be used. The $t$ test (two-tailed) was used to confirm statistical significance. However, because the changes were in the opposite directions, i.e. the average episode number increased while the average length of episode decreased, the total change in workload after combining the two parameters appeared to be insignificant, with $P=0.299$.

The pattern of episode lengths (percentage of short : medium : long: very long episodes) changed from $54: 25: 19: 2$ pre-triage to $65: 20: 14: 1$ post-triage, respectively. There were also changes in the daytime oncall workload (the number of episodes increasing slightly, the length of episode decreasing and the estimated workload decreasing slightly), but none to statistically significant levels.

\section{Discussion}

The overnight shifts were the most affected by the introduction of the new service. The increased number of episodes at night was counterbalanced by their shorter duration, the change of pattern indicating a move from medium, long and very long episodes towards short ones. The overall effect was an increased night workload in terms of time worked per shift (pre-triage average of 190 min per night shift compared with a post-triage average of $243 \mathrm{~min}$ ). The most likely explanation for this change is an increase in telephone consultations (usually a 'short' episode), for example between the nurse and the doctor, and a decrease in actual clinical work done by the doctor for some patients (the rest being done by the nurse). However, other factors may also be at play. These might include an increase in referrals to the 'new' psychiatric on-call service. Also, the triage nurse might have had different thresholds for admission from the doctors (e.g. post-triage there were 121 psychiatric admissions from 74242 accident and emergency patient attendances, compared with 96 from 74040 pre-triage).

Table 1. Changes in workload following triage service introduction

\begin{tabular}{|c|c|c|c|}
\hline & Junior doctors' shift & Pre-triage introduction & Post-triage introduction \\
\hline Number of episodes per shift $(A)$ : mean $(95 \% \mathrm{Cl})$ & $17.00-09.00$ & $4.8 *(4.4-5.3)$ & $8.4^{*}(6.8-10.0)$ \\
\hline \multirow[t]{2}{*}{ Length of episode, $\min (B)$ : mean $(95 \% \mathrm{Cl})$} & $17.00-09.00$ & $39.3^{*}(35.0-43.6)$ & $29.0 *(25.4-32.7)$ \\
\hline & Weekend (24 h) & $35.5(31.1-39.8)$ & $29.2(24.2-34.1)$ \\
\hline Total estimated workload per shift, $\min (A \times B)$ & $17.00-09.00$ & $190 * *$ & $243^{* *}$ \\
\hline
\end{tabular}

${ }^{*} P<0.001,{ }^{*} P<0.299$ 
The difference is unlikely to be due to 'settling in' of the service or seasonal variation, given the time frames used. An analysis of missing or inaccurate data input sheets showed these to be randomly distributed, meaning systematic bias was not a cause.

The reduction in daytime workload, although not reaching statistical significance, may represent a better 'mopping up' of problems the night before, leaving less to be done the next day. However, it could equally represent work that might previously have been left to the next day, being done out-of-hours instead, albeit shared between the nurse and doctor.

These results must be interpreted in the light of the whole service model at the time of study, especially the relative lack of alternatives to admission and the limited nature of the triage service itself (limited hours, competing duties). A more comprehensive and focused triage service might well obtain quite different results, especially if part of a coordinated package including viable alternatives to admission. This is an important point, as in the present financial climate mental health providers may be tempted to cut corners in their search for ways to reduce junior workloads. However, this study implies that a less than comprehensive and coordinated approach might be at best a waste of resources, at worst a step backwards, at least in terms of improving juniors' working hours. Some more ambitious alternatives are being piloted in mental health services elsewhere by the government but only in one site for out-of-hours work, while the legally driven time scale is pressing hard. It is likely that most service providers will have to plan their immediate workforce changes before the results are clear. Our study implies that simply adding a limited triage service alone is insufficient to reduce hours, and that whatever solutions are attempted, the resultant changes should be monitored, rather than taken for granted.

With regard to patient and staff satisfaction, anecdotally both patients and staff (nursing and medical) liked the new triage service, but unfortunately we did not formally measure this. There was no complaint related to the new service, and the annual number of formal complaints for the whole unit decreased from 36 (pretriage) to 15 (during triage), implying the new service probably had not had a detrimental effect on quality, and had possibly improved it.

\section{Acknowledgements}

We thank the junior doctors at Leighton Hospital, Crewe, who filled out all the data forms while on shift, and $\operatorname{Dr} C$. Findlay and Dr R. Hodgson, consultant psychiatrists, for comments on the script, encouragement and guidance. Thanks also to Hillary Hall who input the data. The Mid Cheshire Hospitals NHS Trust sponsored the study.

\section{Declaration of interest}

None.

\section{References}

CASS, H. D., SMITH, I., UNTHANK, C., et Judgement of 3 October 2000: Case Cal (2003) Improving compliance with requirements on junior doctors' hours. $B M J, 327,270-273$.

COUNCIL DIRECTIVE (1993) Council Directive 93/104/EC. Official Journal of the European Community, L307, $18-24$. 303/98 Sindicato de Medicos de Asistencia Publica (SIMAP) v Conselleria de Sanidad. European Court Reports, 2000, 1-7963.

NATIONAL HEALTH SERVICE MANAGEMENT EXECUTIVE (1991) Junior Doctors: The New Deal. London:

DEPARTMENT OF HEALTH (2000) A General Guide to the New Pay System. London: Department of Health. Department of Health

SPSS (1999) SPSS Software: Release 9.0. Chicago, IL: SPSS Inc.

*A. P. Moore Specialist Registrar in General Adult Psychiatry, Lyme Brook Mental Health Resource Centre, Talke Road, Newcastle-under-Lyme, Stoke-onTrent ST57TL, S. Willmott Statistician, Leighton Hospital, Crewe, Cheshire 